\title{
Qualitative Model for Risk Assessment in Construction Industry: A Fuzzy Logic Approach
}

\author{
Abel Pinto ${ }^{1}$, Isabel L. Nunes ${ }^{1}$, and Rita A. Ribeiro ${ }^{2}$ \\ ${ }^{1}$ Universidade Nova Lisboa / FCT, Caparica, 2829-516 Portugal \\ abel.fnpinto@gmail.com, imn@fct.unl.pt \\ ${ }^{2}$ Uninova, Campus UNL/FCT, Caparica, 2829-516 Portugal \\ rar@uninova.pt
}

\begin{abstract}
Risk Assessment for Health and Safety (RAH\&S) of workers is a complex task that entails the consideration of many parameters which are, more often than not, difficult to quantify. RAH\&S in the construction industry is rampant with inadequate data and/or imprecise and incomplete information, particularly in the design stage, for which traditional quantitative approaches do not give adequate answers. In this work we outline the basic aspects for a Qualitative Risk Assessment Model (QRAM) based on elicited data and using a fuzzy logic approach.
\end{abstract}

Keywords: Risk assessment, safety, fuzzy sets, construction industry.

\section{Introduction}

Safety is a prominent feature to be taken into account in the construction industry, and Risk Assessment for Health and Safety (RAH\&S) is a key step to achieve it, particularly to support decision-making in safety programs (Ringdahl, 2001).

In general, risk assessment is a complex process that entails the consideration of many qualitative parameters which are difficult to quantify. Risk assessment in the construction industry, due the specific nature of the sector (Dedobbeleer e Beland, 1991; Ringen et al., 1995; Gillen et al, 1997; Laitinen, 1999; Loosemore, 2001; Tam et al., 2004), must deal with ill-defined and imprecise data and information. So far traditional approaches do not seem to provide adequate answers (Ringen et al., 1995) to deal with this issue. Moreover, using probability (classical or Bayesian) or statistics theories may mask other aspects of incomplete and imprecise knowledge and can lead to a false sense of accuracy and precision, thus leading to inadequate and/or inefficient decisions.

To overcome some of the mentioned problems in assessing safety risks, we propose a qualitative model for risk assessment, hereinafter denoted QRAM (Qualitative Risk Assessment Model), which based on elicited data and using a fuzzy logic approach. The goal is to contribute to work safety by improving risk assessment in construction sites. 


\section{Contribution to Technological Innovation}

The majority of quantitative traditional methods use probabilistic techniques and / or statistics to deal deal with the intrinsic uncertainty and imprecision in data and information. This implies some limitations, such as requiring analysts to estimate parameters, to ensure a sufficiently representative domain or make comparisons with other sites (which departs from the real system under study).

Recently, there are also qualitative approaches using fuzzy logic, proposed in the literature (see for example: Azadeh et al., 2008, Gurcanli, Mungen, 2009), but they lack having a systematic survey of all parameters that should be taken into consideration, together with a general formulation to obtain a complete risk assessment measure.

Hence, the main contributions of the proposed QRAM are: to provide a framework for factors that need to be evaluated in construction sites; to propose a formula expressing the relationships between the parameters (risk factors), thus obtaining an overall risk measure; to provide a simple semantic scale to facilitate the elicitation of information about the parameters. The final aim of QRAM is to obtain an overall measure of risk for the construction site as well as to obtain partial risk calssifications for important parameters included in the overall measure.

QRAM will focus on elicited data, thus avoiding estimates to allow assessment of actual risks of the site. The data and information will be obtained by direct observation, interviews with workers and foremen and also from consultation and review of documentation of the site (health and safety plan, reporting accidents and incidents, records of meetings, work procedures...), among others. After we transform the collected data, using fuzzy logic techniques, and then aggregate it to obtain the risks level classification for the site.

\section{Background on Risk Assessment for Health and Safety (RAH\&S)}

Traditional RAH\&S methods are typically based on information, which is subject to uncertainty (usually handled by probability and/or statistics theories) or is just illdefined. Due to its intrinsic uncertainty and incompleteness traditional RAH\&S methods present some limitations, as pointed by some authors [Karwowski \& Mital, (1986); Cornell (1996); Wang et Ruxton. (1997); Pender (2001); Sii et al. (2001); Tixier (2002); Faber (2003); Nilsen (2003); Kentel et Aral. (2004)], such as:

○ Inherent imprecision in human-centered systems;

- Difficult to generate a mathematical model due to intrinsic uncertainty in this type of problems. For instance, it is difficult to represent and describe the safety behavior, which causes work accidents involving operational procedures, human error and decisions taken by designer and management;

- Difficult to quantify the effects and consequences of hazards because they involve many factors with a high level of uncertainty, even when the physical processes are clearly understood; 
- Large number of assumptions, judgments and opinions are involved in a risk quantification process, hence, it requires considerable skills from a safety analyst to interpret results;

- Construction projects are unique by definition. This reduces the relevance and reliability of statistical aggregates derived from probability-based analysis;

- Humans are limited in their ability to encompass and process the full range of information required for holistic decisions;

- Uncertainty and ignorance may be found in temporal aspects of the flow of knowledge, which are important in project planning;

o Project parameters and outcomes must be communicated to others and the imprecision of our language is not good to express these.

Considering all above statements, it is apparent that RAH\&S in the construction industry is rampant with inadequate data and/or imprecise, ill-defined, and incomplete information, particularly in the design stage, for which traditional approaches do not give adequate answers. Fuzzy logic (Zadeh, 1965, Zimmerman, 1991) provides a natural way of modelling the intrinsic vagueness and imprecision of subjective assessments, while also allows the inclusion of human creativity and intuition, which is an essential ingredient for successful risk analysis (Ru, 1996). However, most qualitative approaches neither consider a systematic framework for all parameters that can influence the risk nor formalize the relationships between parameters (Liu et al., 2001, Tixier et al., 2002, Mure et al., 2006).

\section{Proposed Qualitative Model: QRAM}

In this section we outline the basic aspects that a model for RAH\&S in the construction industry should account for. The knowledge acquisition (i.e. data elicitation) should be obtained by observation of reality, interviews with workers, foreman and engineers, responsible and consultation of site documents (working procedures, reports of work accident investigation ....). The collected data shall be transformed using fuzzy sets (Azadeh et al., 2008) and will be aggregated with specialized fuzzy operators, to obtain a ranking of risks in the construction site.

The starting point is the following formulation for risk assessment in construction sites, $R A C$, as shown in eq. 1.The risk is directly proportional to organizational inadequacies $\left(O_{I}\right)$, which are not related with a specific hazard, and other direct factors $\left(F_{D}\right)$, and additional factors $\left(F_{A}\right)$. The direct and additional factors are inversely proportional to safety barriers factors $\left(S_{B}\right)$, which are also dependent on some hazard.

$$
R A C=O_{I} \otimes \frac{F_{D} \oplus F_{A}}{S_{B}}
$$

$F_{D}$ are the main direct factors related with the hazard under analysis (for example, in falls from height, the factor is the height of the fall). 
$F_{A}$ are additional factors; they also depend on hazards under analysis and include a variable number of parameters. Examples of aditional factors (for the same hazard) are: tidiness of workplace, inclination of work floor, friction level between shoe's soles and soil.

Both direct factors and additional factors include a variable number of parameters, which will depend on the site being evaluated. The risk specialist will choose which factors will be evaluated for the specific site. In general the formulation will be:

$$
F_{(D \cup A)}=\oplus\left(f_{1}, f_{2}, \ldots, f_{n}\right) / N
$$

Where $\mathrm{N}$ is the number of parameters that will be considered for any of the two factors.

$O_{I}$ express organizational inadequacies and are a function of the lack (or poor): safety culture $\left(S_{c}\right)$, safety organisation $\left(S_{o}\right)$, work organisation $\left(W_{o}\right)$, supervision $(S)$, leadership ( $L$ ), personal factors and communications $(C$ ). Formally,

$$
O_{I}=\oplus\left(S_{c}, S_{o}, W_{o}, S, L, P, C\right) / N
$$

with $\mathrm{N}$ being the number of parameters.

$S_{B}$ represent the Safety Barriers implemented in the site for controling the risk. Their effectiveness should be measured by:

$$
S_{B}=\oplus(M, A, S, I) / N
$$

where ( Hollnagel 1999):

○ $\quad M$-Material barriers physically prevent an action from being carried out or the consequences from spreading;

o $A$-Functional (active or dynamic) barriers work by impeding the action to be carried out, for instance by establishing a logical or temporal interlock.

- $\quad S$-Symbolic barriers require an act of interpretation in order to achieve their purpose, hence an "intelligent" agent that can react or respond to the barrier.

o I-Immaterial barriers are not physically present or represented in the situation, but depend on the knowledge of the user to achieve their purpose.

The proposed process to obtain the final RAH\&S measure, $R A C$ (eq 1), will use concepts and techniques from fuzzy set theory (Zadeh, 1965, 1975, 1983).

In this first work we use a single discrete fuzzy set for classifying the factors and their respective parameters. The proposed fuzzy classification can be used to elicit classifications from different sources (e.g. workers, engineers, safety experts), for all parameters in a user-friendly semantic form. Formally, the general fuzzy parameter classification membership function, to be used in this preliminary model is:

$$
\text { ParameterClassification }=\left\{\begin{array}{l}
\text { Excelent } / 1, \text { VeryGood } / 0.8, \text { Good } / 0.6, \\
\text { Satisfactory } / 0.4, \text { Mediocre } / 0.2, \text { Bad } / 0.001
\end{array}\right\}
$$

At this stage, the terms and respective values considered are just indicative and in future work they will be validated and tuned. All parameters in eq. 2, eq. 3 and eq. 4 
(e.g. material barriers $M$ ) will be classified using the above discrete fuzzy set. To obtain the values for the respective factors (e.g. $S_{B}$ ) we plan to use an aggregation operator, $\oplus$, such as arithmetic sum, average or any other compensatory operator (Zimmerman, 1991). Finally to obtain the overall risk measure for the construction site, we plan to use conventional arithmetic operators.

At this stage of the research work we are not yet sure about which operators are more suitable for obtaining the risk assessment in the construction industry, but a study about them is foreseen in the near future. Here, as mentioned, we just focused on the conceptual model for determining the risk assessment.

\section{Illustrative Example}

In order to illustrate the applicability of the method, we will use a simple illustrative case for the risk that causes more deaths in Portugal, in the sector: falls from height. In this example we only describe the calculations for additional factors, $F_{A}$, because the process is similar for the other factors in eq. 1.

Consider a construction work on a scaffold with three meters high, thus the single direct factor, is $F_{D}=0.001$, because we assumed the intervals: [0 to $\left.0,5 \mathrm{~m}\right] F_{D}=1$; ]0,5 to $\left.1 \mathrm{~m}] F_{D}=0.8 ;\right] 1$ to $\left.\left.1,4 \mathrm{~m}\right], F_{D}=0.6 ;\right] 1,4$ and $\left.\left.1,6 \mathrm{~m}\right] F_{D}=0.4 ;\right] 1,6$ to 1 , $\left.8 \mathrm{~m}] F_{D}=0.3 ;\right] 1,8$ to $\left.2 \mathrm{~m}\right] F_{D}=0.2$, and $>2 \mathrm{~m} F_{D}=0.001$.

The additional factors considered and the respective semantic classification, using the fuzzy membership semantic variables (eq. 5) are: (a little clean and tidy) $=$ mediocre $;($ horizontal surface $)=$ good $;($ foot boards, wood, presented in good repair $)=$ satis factory; (when dry, the material floor provides good traction on the sole of the shoe) = satisfactory; (lighting level well suited to work) = good, (irons waiting on the surface of the collision $)=b a d$; $($ dry weather and no wind $)=\operatorname{good}($ scaffolding meet all legal and regulatory requirements, was well fitted and properly anchored $)=\operatorname{good}$.

Now, aggregating the classifications for the additional factors (eq. 2), using the membership values of eq. 5 , we have:

$$
F_{A}=(0.2+0.6+0.4+0.4+0.6+0.001+0.6+0.6) / 8=0.4375
$$

Notice that in this example we used arithmetic operators to aggregate the values, but in the future other operators will be tested to improve the method.

Although the six factors are appropriate, in various degrees: good (4) and satisfactory (2), there are two factors with inappropriate levels, one mediocre and another bad. Hence, the combination is only satisfactory (closest semantic level in the classification fuzzy set for the result 0.4375). This result means the site safety has to be improved, particularly to protect the irons on hold (bad) and clean and organize the workplace (mediocre).

Regarding the safety barriers $S_{B}$, the scaffold has railings around the outside perimeter of the platforms, properly fitted, good and robust (very good), the inner side is $20 \mathrm{~cm}$ from the construction (very good), the access to work platforms is appropriate and also well protected (very good). The company implemented a procedure 
for working at heights which includes work on scaffolding, the procedure is well prepared and written in language appropriate to their users (very good). The company provides training to all workers on scaffolding, however, the training records do not show the knowledge acquired by trainees is properly understood, hence the company did not prove the effectiveness of training (mediocre). Following the above calculation for additional factors and using eq. 4 ,

$$
S_{B}=(0.8+0.8+0.8+0.8+0.2) / 5=0.68
$$

This result shows that the efficiency in the safety barriers is good (closest semantic value for the result 0.68 ).

Regarding the organizational factors $O_{I}$ (eq. 2) and for simplicity we consider an overall classification of excelent (membership value $=1$ ).

Finally we can determine the level of risk assessment for the example, following eq. 1 ,

$$
R A C=1 \times \frac{0.1+0.4375}{0.68}=0.79
$$

Notice we used again arithmetic operators to improve clarity of method' explanation. The final risk level in the construction site for the falling height, yields a value of very good (corresponding to the closest semantic variable).

Although the criteria for acceptability of risk is appropriate and very good there is room for improvements, namely implement new safety barriers and also improve some additional factors (e.g. improve some aspects related to training).

\section{Conclusion and Further Work}

Fuzzy approaches for human-centred problems seem to be quite flexible, hence in this work we introduce a preliminary version of a qualitative method for RAH\&S, in the construction industry, using fuzzy logic concepts and techniques. From all pointed limitations and inadequacies of traditional RAH\&S methods it seems that using this type of fuzzy approach, to evaluate work safety factors, yields a more realistic representation and solution for evaluation of risks in the construction industry.

The outlined qualitative model (QRAM) is still under development. It needs to be further improved and refined, such as identifying other types of hazards and further characterize all risks involved, as well as to set criteria for the tolerability of risk analysis in construction sites. After these improvements it needs to be tested and validated with real case studies to assess its suitability and generality for the construction sector.

\section{References}

1. Azadeh, A., Fam, I.M., Khoshnoud, M., Khoshnoud, M.: Design and implementation of a fuzzy expert system for performance assessment of an integrated health, safety, environment (HSE) and ergonomics system: The case of a gas refinery. Information Sciences 178, 4280-4300 (2008) 
2. Cornell, M.E.P.: Uncertainties in risk analysis: Six levels of treatment. Reliability Engineering and System Safety 54, 95-111 (1996)

3. Dedobbeleer, N., e Beland, F.: A Safety Climate Measure for Construction Sites. Journal of Safety Research 22, 97-103 (1991)

4. Faber, M.H., Stewart, M.G.: Risk assessment for civil engineering facilities: critical overview and discussion. Reliability Engineering and System Safety 80, 173-184 (2003)

5. Gurcanli, G.E., Mungen, U.: An occupational safety risk analysis method at construction sites using fuzzy sets. International Journal of Industrial Ergonomics 39, 371-387 (2009)

6. Hollnagel, E.: Accidents and barriers. In: Proceedings of the European Conference on Cognitive Science Approaches to Process Control, CSAPC (1999)

7. Karwowski, W., Mital, A.: Potential Applications of Fuzzy Sets in Industrial Safety Engineering. Fuzzy Sets and Systems 19, 105-120 (1986)

8. Kentel, E., Aral, M.M.: Probabilistic-fuzzy health risk modeling. Stoch Envir. Res. and Risk Ass. 18, 324-338 (2004)

9. Laitinen, H., Marjamaki, M., e Paivarinta, K.: The validity of the TR safety observation method on building construction. Accident Analysis and Prevention 31, 463-472 (1999)

10. Liu, J., et al.: Fuzzy Rule-Based Evidential Reasoning Approach for Safety Analysis. International Journal of General Systems 33(2-3), 183-204 (2004)

11. Loosemore, M., e Lee, P.: Communication problems with ethnic minorities in construction industry. International Journal of Project Management 20, 517-524 (2001)

12. Mure, S., Demichela, M., Piccinini, N.: Assessment of the risk of occupational accidents using a fuzzy approach. Cogn. Tech. Work 8, 103-112 (2006)

13. Nilsen, T., Aven, T.: Models and model uncertainty in the context of risk analysis. Reliability Engineering and System Safety 79, 309-317 (2003)

14. Pender, S.: Managing incomplete knowledge: Why risk management is not sufficient. International Journal of Project Management 19, 79-87 (2001)

15. Ringdahl, L.H.: Safety Analysis principles and pratice in occupational safety, 2nd edn. Taylor \& Francis, London (2001)

16. Ringen, K., Englund, A., Welch, L., Weeks, J.L., Seegal, J.L.: Why construction is different. Occupational Medicine 10, 255-259 (1995)

17. Ru, W.G., Eloff, J.H.P.: Risk analysis modelling with the use of fuzzy logic. Computers \& Security 15(3), 239-248 (1996)

18. Sii, H.S., Wang, J., Ruxton, T.: Novel risk assessment techniques for maritime safety management system. International Journal of Quality \& Reliability Management 18(8/9), 982999 (2001)

19. Tam, C.M., Zeng, S.X.E., Deng, Z.M.: Identifying elements of poor construction safety management in China. Safety Science 42, 569-586 (2004)

20. Tixier, J., et al.: Review of 62 risk analysis methodologies of industrial plants. Journal of Loss Prevention in the Process Industries 15, 291-303 (2002)

21. Wang, J., Ruxton, T.: A review of safety analysis methods applied to the design process of large engineering products. J. Eng. Design 8(2), 131-152 (1997)

22. Zadeh, L.A.: Linguistic variables, approximate reasoning and dispositions. Med. Inform. 8(3), 173-186 (1983)

23. Zadeh, L.A.: The concept of a linguistic variable and its application to approximate reasoning-part I. Information Sciences 8, 199-249 (1975)

24. Zadeh, L.A.: Fuzzy sets. Information and Control 8, 338-353 (1965)

25. Zimmerman, H.J.: Fuzzy Set Theory and its Applications, 2nd edn., pp. 36-43. Kluwer Academic Publishers, London (1991) 